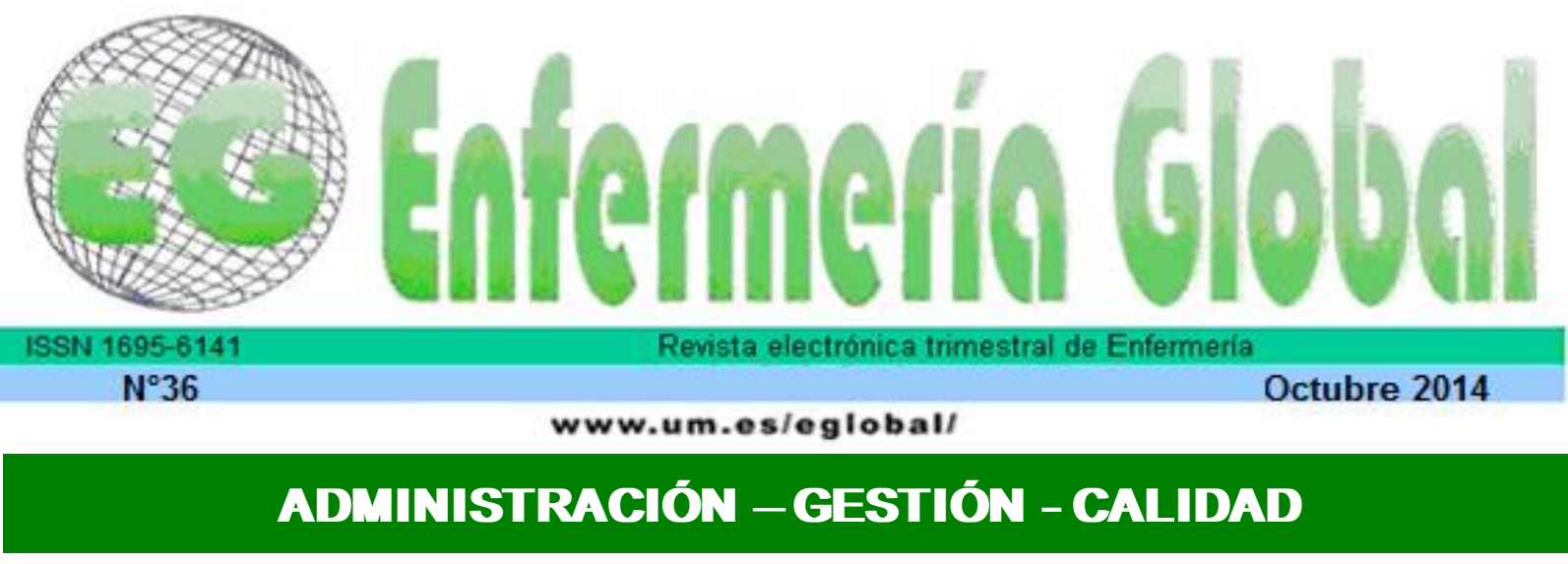

\title{
Prevalencia del Síndrome del Burnout en el personal de enfermería del Servicio de Cardiología, Neumología y Neurología del Hospital San Jorge de Huesca
}

Prevalence of burnout syndrome in nurses of the Cardiology, Pneumology and Neurology, Hospital San Jorge of Huesca

\section{"Sanjuan Estallo, Laura ${ }^{* *}$ Arrazola Alberdi, Oihana ${ }^{* * *}$ García Moyano, Loreto María}

*Diplomada Universitaria en Enfermería. **Máster em Ciencias de la Enfermería. Enfermera del Servicio de Cardiología, Neumología y Neurología del. Hospital San Jorge. Huesca. ${ }^{* * *}$ Docente Grado de Enfermería Universidad San Jorge. Zaragoza. E-mail: loretongarcia@hotmail.com

Palabras clave: Síndrome de Burnout; Enfermería; Maslach Burnout Inventory

Keywords: Burnout Syndrome; Nursing; Maslach Burnout Inventory

\section{RESUMEN}

El objetivo fue determinar la prevalencia del Síndrome del Burnout en el personal de enfermería del Servicio de Cardiología, Neumología y Neurología del Hospital San Jorge de Huesca. Se realizó un estudio descriptivo transversal, las variables sociodemográficas se recogieron mediante un cuestionario de elaboración propia, el Síndrome de Burnout se midió mediante el Maslach Burnout Inventory, en su versión validada en español. El total de los participantes fue de 22. La edad media fue de 40,8 años. El $36,4 \%$ trabaja en el servicio de Cardiología, el 31,8\% en Neumología y el 31,8\% en Neurología (Área de Ictus). La prevalencia del Síndrome de Burnout fue del $31,81 \%$. No se encontraron diferencias estadísticamente significativas de padecimiento del síndrome según la edad, ni entre el personal de enfermería dedicado al servicio de Neurología (Área de Ictus) y resto de unidades $(p=0,630)$.

\section{ABSTRACT}

The objective was to determine the prevalence of burnout syndrome in nurses of the Cardiology, Pneumology and Neurology, Hospital San Jorge of Huesca.We performed a cross-sectional study, the sociodemographic variables were collected using a specifically designed questionnaire, the burnout syndrome was measured by the Maslach Burnout Inventory in its validated Spanish version. The total number of participants was 22. The average age was 40.8 years, $36.4 \%$ work in the service of Cardiology, $31,8 \%$ of Pneumology and $31,8 \%$ of Neurology (Ictus Area).No significant statistically differences were found of syndrome disease by age or between nursing staff dedicated to serving Neurology (Ictus Area) and other units $(p=0.630)$. 


\section{INTRODUCCIÓN}

La organización mundial de la salud define estrés como: "El conjunto de reacciones fisiológicas que prepara el organismo para la acción”. El estrés, forma parte de nuestra vida moderna, afectando a la salud, bienestar psicológico y rendimiento profesional $^{(1)}$.

En lo que al estrés laboral se refiere, surge el "Síndrome del Burnout" cuya traducción correspondería a "síndrome de estar quemado". Este constructo que engloba diversos signos y síntomas, ha llegado incluso a plantearse como enfermedad profesional ${ }^{(2,3)}$.

Fue en 1974 cuando el término "Burnout" fue descrito por Herbert Freudemberguer, psicoanalista, quien observo distintas manifestaciones como ansiedad, depresión y agotamiento en psicoterapeutas que pretendían modificar la conducta de los pacientes. Parece ser que esto les convertía en profesionales más insensibles y poco comprensivos. Fue entonces cuando definitivamente definió el concepto del síndrome ${ }^{(4,5,6)}$.

Siguiendo esta línea, en 1981, Maslach y Jackson, especialistas en psicología de la salud, pasaron a definir este concepto como "síndrome de agotamiento emocional, despersonalización y baja realización profesional, que puede ocurrir entre individuos que trabajan con personas" ${ }^{(7)}$. Se consideró esta definición multidimensional, ya que además, se confirmó que el síndrome iba acompañado de síntomas físicos. Identificando estas características se pudo crear una escala, denominada Maslach Burnout Inventory (MBI) que engloba tres subescalas: cansancio emocional (CE), despersonalización (DP) y falta de realización personal $(\mathrm{RP})^{(8,9,10,11,12)}$.

Actualmente, se han determinado diferentes factores profesionales, institucionales y personales relacionados con la aparición del síndrome ${ }^{(2)}$. Los factores de riesgo relacionados especialmente con el personal sanitario son:

- Factores ambientales (sobrecarga de trabajo, presión de tiempo, insatisfacción laboral, falta de recompensas profesionales, contacto con el sufrimiento y la muerte, relaciones difíciles con los pacientes y sus familiares, insuficientes recursos materiales, falta de autonomía, etc.) ${ }^{(5,13)}$.

- Factores personales (carácter, temperamento, estrategias de afrontamiento, supresión de pensamientos, mecanismos para mejorar la inteligencia emocional, etc.) $(5,13)$

- Factores de relación con el paciente (problemas de interacción, comunicación insuficiente, etc.) ${ }^{(5)}$.

Así mismo, a nivel fisiológico las consecuencias podrían ser falta de apetito, cansancio, insomnio, dolores de cabeza, agotamiento, etc.; a nivel psicológico; cambios de actitud, irritabilidad, ansiedad, sentimiento de frustración, etc., y a nivel conductual; hostilidad, incapacidad de concentración, conflictos, etc.

A todo esto debemos sumar los no menos relevantes, costes económicos; disminución del rendimiento laboral, incremento de la accidentalidad, absentismo laboral, etc. En Europa se ha cifrado el coste total anual en torno a 20.000 millones de 
euros por razones de absentismo o incapacidad temporal relacionadas con el estrés laboral (14).

Actualmente, la expresión Burnout se utiliza para hacer referencia a un tipo de estrés laboral crónico, generado específicamente por profesionales que mantienen una relación y contacto directo con personas, especialmente en relaciones de ayuda. Por lo que afecta sobre todo a colectivos como profesores, policías, personal de salud, etc. ${ }^{(11,15,16,17)}$.

En cuanto al colectivo que nos ocupa, los profesionales de la salud, existe amplia bibliografía que demuestra su mayor vulnerabilidad a padecer este síndrome. De hecho, este grupo, en especial los profesionales enfermeros (por su alto grado de relación con el paciente), ha sido identificado como uno de los grupos con mayor riesgo de desarrollar el síndrome ${ }^{(18,19,20)}$.

En los estudios dedicados a enfermería, se ha identificado más prevalencia en edades altas, así como en enfermeros que trabajan en áreas de cuidados más específicos e intensivos. Según estos estudios, en dichos servicios existen más condiciones que pueden influir en el padecimiento como: las características del trabajo, la tecnificación del ambiente laboral, las demandas emocionales y físicas, la gravedad del estado de los pacientes tratados, la angustia e incertidumbre de familiares e incluso, el proceso de muerte y duelo ${ }^{(4,21,22,23)}$. Se consideran factores predisponentes tanto la exposición al sufrimiento y al final de la vida, como la insuficiente formación de los profesionales en el manejo de estas situaciones ${ }^{(24)}$. Esto lógicamente, se ha relacionado con un gran desgaste físico y psicológico de la persona que cuida, y en consecuencia, esto influye en la atención individualizada, holística e integral que la profesión requiere.

En el Servicio de Cardiología, Neumología y Neurología del Hospital San Jorge de Huesca, Servicio hasta 2010 dedicado principalmente a las especialidades de Cardiología y Neumología, surge una nueva Área especializada para el cuidado del paciente afectado por Ictus (englobada dentro del programa CODIGO ICTUS). En el proceso de instauración del programa fue necesario un aumento en la plantilla de enfermería, cambios estructurales, formación para el personal, etc. En consecuencia se introdujeron tareas y cuidados más concretos y constantes por parte del personal enfermero. Por ejemplo; valoración por medio de escalas especializadas, observación constante de la evolución de los pacientes, una permanente monitorización y medición de constantes vitales, etc.

Debido a las graves consecuencias que acarrea el Síndrome del Burnout $(2,12,19,20,21,23,25,26)$, y tras los cambios introducidos en dicho servicio, se planteó la siguiente pregunta de investigación; ¿Qué prevalencia del Síndrome de Burnout existe en el personal de enfermería del Servicio de Cardiología, Neumología y Neurología del Hospital San Jorge de Huesca?. Siendo el objetivo principal, estimar la prevalencia del Síndrome de Burnout en el personal de enfermería del Servicio de Cardiología, Neumología y Neurología del Hospital San Jorge de Huesca y asimismo, conocer la distribución del padecimiento o no padecimiento del síndrome según el sexo, la edad y el área de trabajo a la que pertenecían. 


\section{METODOLOGÍA}

Estudio observacional, descriptivo, transversal y retrospectivo. La población a estudio estuvo compuesta por los profesionales de enfermería que trabajaban en el servicio de Cardiología, Neumología y Neurología del Hospital San Jorge de Huesca en los meses de febrero y marzo de $2013(\mathrm{~N}=24)$. Se consideraron criterios de inclusión, personal fijo, interino o suplente, que trabajara en dichos servicios en el tiempo que duró la investigación de manera continuada y en condiciones de turnicidad (turnos de mañana, tarde y noche), se consideró criterio de exclusión, trabajadores que contaban con un contrato a jornada reducida, 2 trabajadores en total $(8,33 \%$ del total de la plantilla) por no estar expuestos a los factores de riesgo durante el mismo tiempo que el resto. El número total de enfermeros a estudio fue de 24, resultando finalmente incluidos en el estudio 8 personas pertenecientes al Servicio de Cardiología, 7 trabajadores del Servicio de Neumología y 7 del Servicio de Neurología (Área de Ictus), formados especialmente para desempeñar su trabajo en dicha área ( $\mathrm{N}=22)$.

De manera general se pude afirmar que los pacientes usuarios de los servicios de Neumología y Cardiología de dicho Hospital tienen el siguiente perfil; edad avanzada, con un elevado porcentaje de comorbilidad, una alteración intermedia en la calidad de vida y un nivel moderado de conocimientos sobre el régimen terapéutico y son independientes para actividades de la vida diaria (AVD), mientras que los usuarios del servicio de Neurología suelen tener el siguiente perfil; edad variable, con menor porcentaje de comorbilidad, no alteración previa de la calidad de vida, un nivel moderado de conocimientos sobre el régimen terapéutico y son en el momento de su ingreso más dependientes para actividades de la vida diaria (AVD). Así, los ratios enfermera-paciente eran de 1 enfermera /11 pacientes en el turno de mañana, 1 enfermera/14 pacientes en el turno de tarde y 1 enfermera/19 pacientes en el turno de noche.

Uno de los instrumentos utilizados para la recogida de datos ha sido un cuestionario donde se recogen datos socio-demográficos de la muestra de estudio: sexo, edad y especialidad en la que se trabajaba Cardiología, Neumología o Neurología (Área de Ictus).

El segundo instrumento de recogida de datos ha sido el cuestionario Maslach Burnout Inventory (MBI) en su versión validada en español $(27,28)$. Las tres subescalas han alcanzado valores de fiabilidad alfa de Cronbach aceptables (0,90 para agotamiento emocional, 0,79 para despersonalización y 0,71 para realización personal en el trabajo). El cuestionario consta de 22 ítems valoradas en una escala tipo likert con cuatro posibles respuestas, y mide las tres dimensiones del síndrome de Burnout $(7,8,27,28)$ :

- Agotamiento emocional, consta de 9 ítems. Valora la vivencia de estar exhausto emocionalmente por las demandas del trabajo. La puntuación máxima es 54.

- Despersonalización, está formada por 5 ítems. Valora el grado en que cada uno reconoce actitudes de frialdad y distanciamiento. La puntuación máxima es 30.

- Realización personal, se compone de 8 ítems. Evalúa los sentimientos de autoeficacia y realización personal en el trabajo. La puntuación máxima es 48 (29). 
Teniendo en cuenta los parámetros establecidos previamente, se categorizan las tres escalas en bajo, medio y alto. Para la dimensión de cansancio emocional: $<19$ (bajo) ,19-26 (medio), >27 (alto), despersonalización: <6 (bajo), 6-9 (medio), >10 (alto) y para la dimensión de realización personal: $<33 \mathrm{~m}$ (bajo), 34-39 (medio), >40 (alto). En caso de obtener un cansancio emocional alto, una despersonalización alta y una baja realización personal, se identifica la presencia del síndrome $(30,31,32)$.

El material se distribuyó entre el personal de enfermería de dichos servicios, durante el mes de febrero y marzo de 2013, en turno de mañana, tarde y noche. Se administraron y recogieron las encuestas personalmente.

Para el registro de las variables a estudio y el tratamiento estadístico, se utilizó el paquete estadístico SPSS V.19. El análisis descriptivo de la muestra se realizó en función de la naturaleza, nivel de medición y escala de cada una de las variables implicadas. Así, las variables categóricas han sido descritas mediante tablas de frecuencias, y las variables cuantitativas mediante medidas de tendencia central (media y mediana) y de dispersión (rango y desviación típica).

El estudio de la asociación estadística entre las variables cualitativas del estudio, según la edad y servicio se realizó mediante el contraste de independencia de (ChiCuadrado), procediendo a la reagrupación de categorías cuando fuese necesario. Al mismo tiempo, cuando se obtuvieron frecuencias mínimas esperadas entre 3 y 5 se utilizó la corrección de Yates y cuando se encontraron frecuencias mínimas esperadas inferiores a 3 se utilizó la corrección de Fisher. La edad fue dividida en dos grupos de edad, utilizando para ello la mediana (38 años). El nivel de significación estadístico para este estudio fue fijado en el $5 \%$.

\section{RESULTADOS.}

Del total de los participantes del estudio ( $\mathrm{N}=22), 21$ sujetos $(95.5 \%)$ eran mujeres y 1 $(4,5 \%)$ hombres.

La edad media fue de 40,8 años, con una mediana de 38 años y una moda de 49 años. Valores extremos de edad de 27 y 58 años. Desviación típica de 9,4 años y rango de 31 (Gráfico 1).

\section{Gráfico 1.}

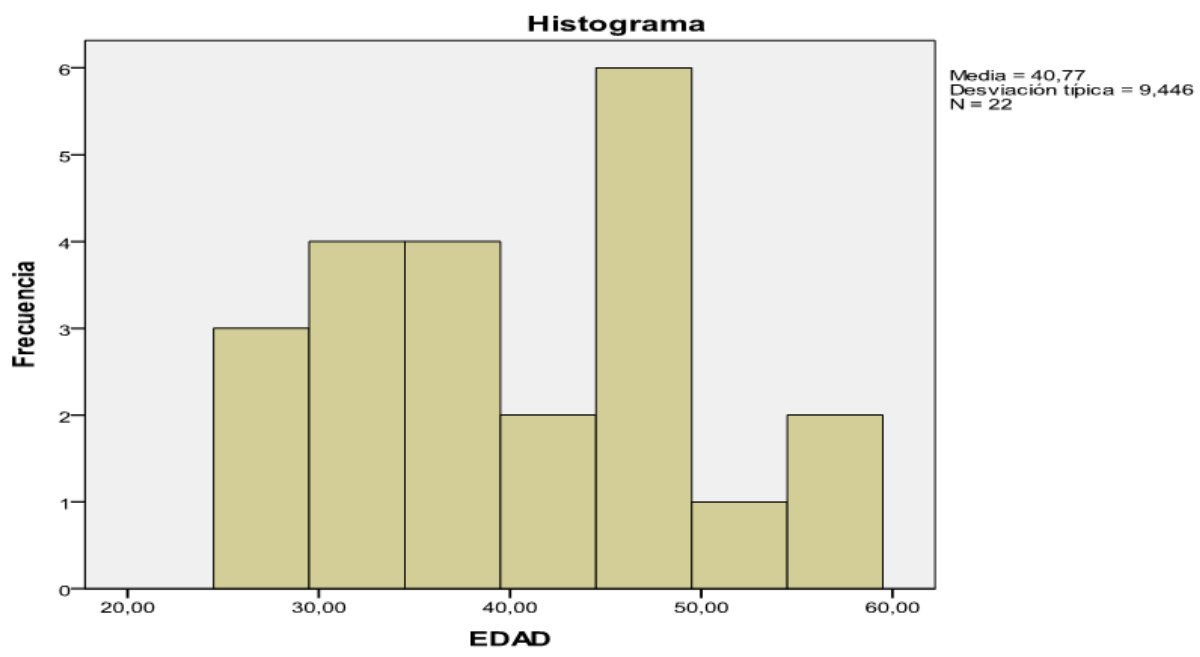


Los resultados obtenidos en las diferentes subescalas del Cuestionario Maslach Burnout Inventory (MBI) fueron los siguientes:

En lo referente al cansancio emocional, que evalúa la sensación emocional de estar cansado por las demandas de trabajo, 9 sujetos (40,9\%) obtuvieron un nivel alto, 2 $(9,1 \%)$ un nivel medio y $11(50 \%)$ un nivel bajo (Gráfico 2$)$.

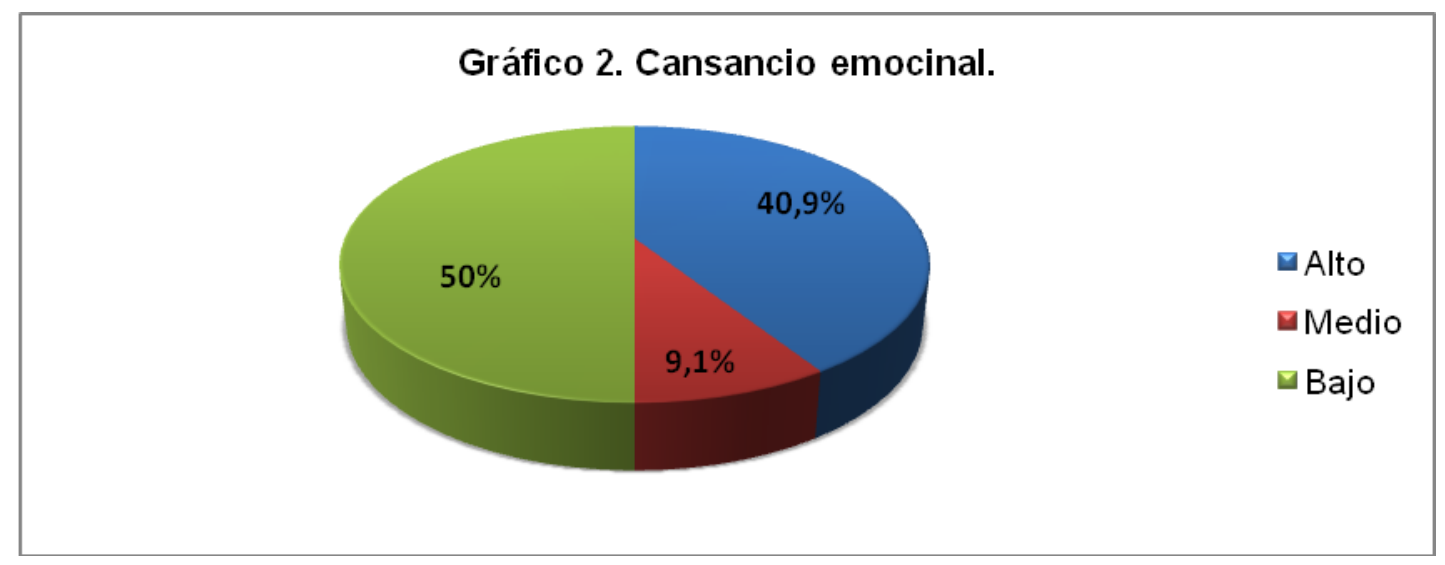

En cuanto a la despersonalización, que evalúa el reconocimiento personal de actitudes de frialdad y distanciamiento, 10 enfermeros (45,5\%) obtuvieron un nivel alto, $5(22,7 \%)$ un nivel medio y $7(31,8 \%)$ un nivel bajo (Gráfico 3).

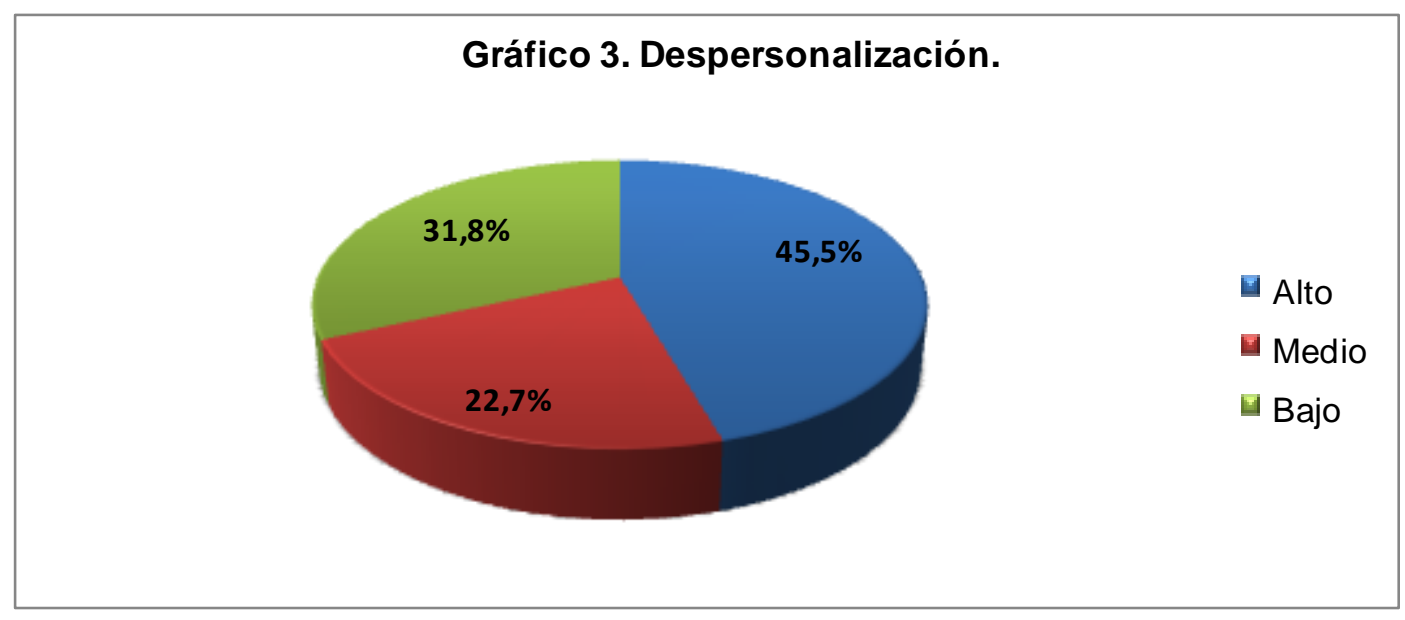

Así mismo, en lo que atañe a la realización personal, es decir, a los sentimientos de autoeficacia laboral, 7 sujetos $(31,8 \%)$ obtuvieron un nivel alto, $2(9,1 \%)$ un nivel medio y $13(59,1 \%)$ un nivel bajo (Gráfico 4$)$. 


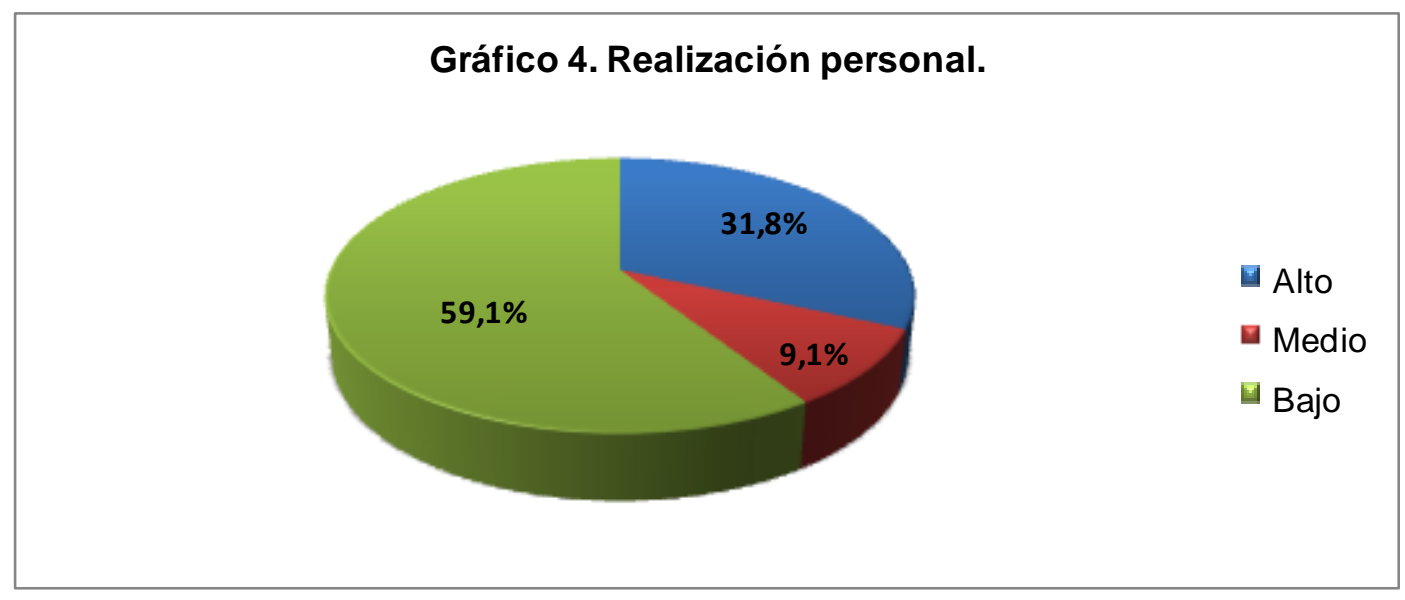

Los resultados finales mostraron una prevalencia del Síndrome de Burnout en 7 sujetos $(31,81 \%)$ con respecto a 15 (68,19\%) que no lo presentaban (Gráfico 5).

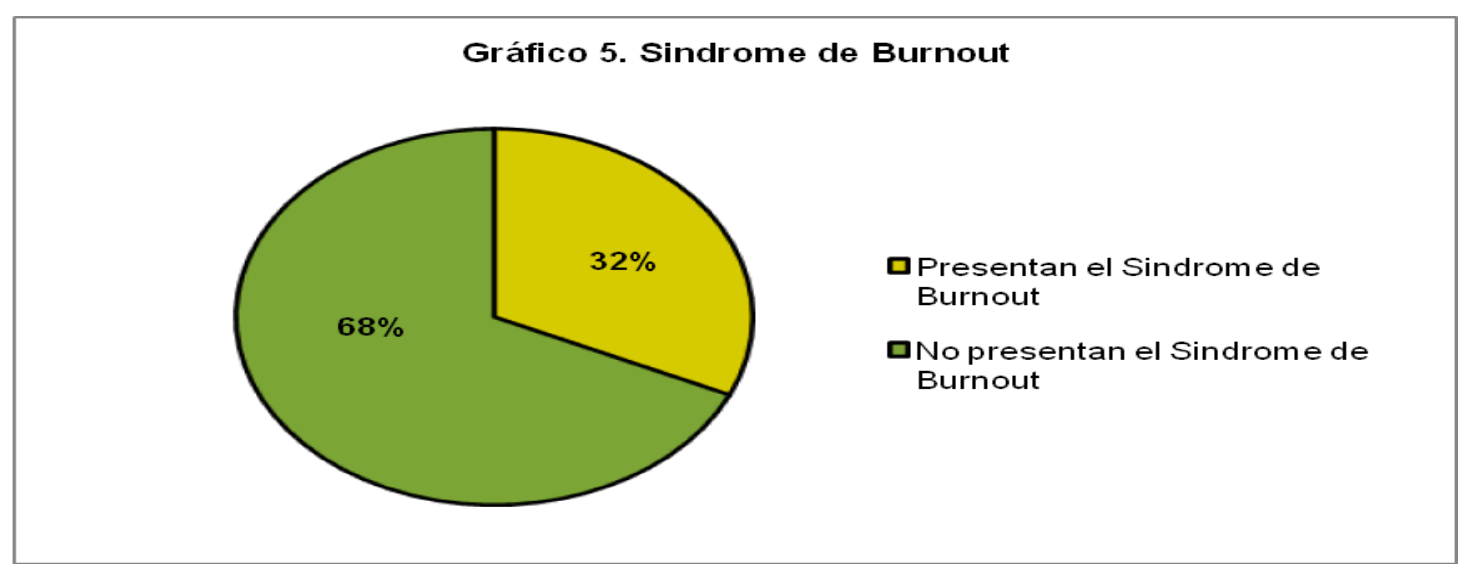

No se encontraron diferencias estadísticamente significativas entre presentar o no presentar el Síndrome de Burnout según la edad, mayores o menores de 38 años $(p=0,067)($ Tabla I).

\begin{tabular}{|c|c|c|c|c|}
\hline \multicolumn{4}{|c|}{ Tabla I. EDAD - S.BURNOUT } \\
\hline \multirow{2}{*}{ Variable } & $\begin{array}{c}\text { Presentan } \\
\text { Burnout }\end{array}$ & $\begin{array}{c}\text { No presentan } \\
\text { Burnout }\end{array}$ & Estadístico \\
\hline \multirow{2}{*}{ Edad } & $\begin{array}{c}\text { Menores de } \\
38 \text { años }\end{array}$ & $1(9,1 \%)$ & $10(90,9 \%)$ & \multirow{2}{*}{$\mathrm{p}=0,067$} \\
\cline { 2 - 4 } & $\begin{array}{c}\text { Mayores de } \\
38 \text { años }\end{array}$ & $6(54,5 \%)$ & $5(45,5 \%)$ & \\
\hline
\end{tabular}

No se encontraron diferencias estadísticamente significativas entre presentar o no presentar el síndrome, según el Servicio de Neurología (Área de Ictus) y el resto de servicios $(p=0,630)$ (Tabla II). 


\begin{tabular}{|c|c|c|c|c|}
\hline \multicolumn{4}{|c|}{ Tabla II. ESPECIALIDAD - S.BURNOUT. } \\
\hline \multirow{2}{*|2}{ Variable } & $\begin{array}{c}\text { Presentan } \\
\text { Burnout }\end{array}$ & $\begin{array}{c}\text { No presentan } \\
\text { burnout }\end{array}$ & Estadístico \\
\hline \multirow{2}{*}{ Especialidad } & $\begin{array}{c}\text { Cardiología } \\
y\end{array}$ & $4(26,7 \%)$ & $11(73,3 \%)$ & \multirow{2}{*}{$\mathrm{p}=0,630$} \\
\cline { 2 - 4 } & $\begin{array}{c}\text { Neumología } \\
\text { Área de } \\
\text { Ictus }\end{array}$ & $3(42,9 \%)$ & $4(57,1 \%)$ & \\
\hline
\end{tabular}

\section{DISCUSIÓN}

En primer lugar, hacemos referencia a la alta participación que hemos obtenido por parte de la muestra del estudio, con una tasa de respuesta del $100 \%$.

En lo que respecta al sexo de la muestra ( $95,5 \%$ de mujeres), los datos encontrados se asemejan a los encontrados en otras bibliografías ${ }^{(21,28,29,33)}$.

En cuanto a los resultados obtenidos para el Síndrome de Burnout y sus dimensiones, se puede decir, que el $31.81 \%$ de la muestra presenta el Síndrome, una cifra relevante y elevada, si se compara con las cifras encontradas en otros estudios como son el de Sánchez Alonso et al. ${ }^{(29)}$ con un 11,7\%, Rodríguez López et al. ${ }^{(34)}$, en el que síndrome de Burnout representa el 10,34\% de la muestra, y el estudio de Ríos ${ }^{(32)}$ en un $12 \%$.

Se puede considerar que la prevalencia encontrada del síndrome, un $31,81 \%$, es una cifra relevante en cuanto en tanto el instrumento de medida utilizado, la escala Maslach Burnout Inventoryes, considerada actualmente el mejor instrumento de medición del burnout, es el más utilizado para las investigaciones relacionadas con este síndrome ${ }^{(35)}$.

Se debe subrayar además, las elevadas cifras halladas en lo que a las dimensiones del síndrome se refiere, ya que el $40,9 \%$ sufre un nivel alto de cansancio emocional, el $45,5 \%$ reconoce tener actitudes de frialdad y distanciamiento con sus pacientes y un $59,1 \%$ tiene un nivel bajo en la consideración de su propia realización personal laboral, datos que nos hacen pensar en que la calidad de los cuidados que proporcionan dichos profesiones a sus pacientes no son los más seguros, ya que existe una relación entre la percepción de la seguridad de los cuidados por parte de los profesionales y el síndrome de burnout, en las dimensiones de agotamiento emocional y despersonalización ${ }^{(36)}$.

En este estudio no se han encontrado diferencias estadísticamente significativas según la edad, mayor o menor de 38 años y el padecimiento del Síndrome. No obstante, como se puede observar en la Tabla I, aparecen diferencias que aun no siendo estadísticamente significativas podrían considerarse como un factor para la aparición del síndrome en aquellas que son mayores de 38 años. Esto coincide con otras bibliografías revisadas donde la edad es un factor determinante $(15,30,31,37)$. Podría considerarse esta observación para futuros estudios y al mismo tiempo introducir otras variables como los años de antigüedad, años trabajados en el servicio, 
estado civil, número de hijos, tipo de contrato, etc. Por ejemplo, Álvarez Verdugo et $\mathrm{al}^{(38)}$ usaron una ficha sociodemográfica que comprendía 18 preguntas, las cuales pertenecían a un grupo de factores psicosociales contenidos dentro de la clasificación de factores de riesgo psicosocial según el Comité Técnico conformado por la OMS y la OIT, o la investigación de Bacaicoa Parrado $\mathrm{P}$, et al. ${ }^{(39)}$ referente al padecimiento del síndrome en unidades de Cardiología, que usaron un cuestionario específico de situaciones concretas que podían ocurrir en un Servicio de Cardiología.

De acuerdo con los resultados obtenidos no se evidencia tampoco asociación entre padecer el Síndrome de Burnout y trabajar en el Servicio de Neurología (Área de Ictus). De hecho, con respecto a las otras especialidades (Cardiología y Neumología) no se han encontrado diferencias en cuanto a la aparición del síndrome. Esta situación no coincide con otros estudios llevados a cabo por otros autores en los que sí se encontraban diferencias estadísticamente significativas entre los grupos de profesionales de enfermería que se dedicaban a áreas más especializadas, como por ejemplo: Pediatría, Oncología y Unidad de de Cuidados Intensivos (UVI) $(2,4,12,15,20,21,26,30,32)$. Diferentes estudios han demostrado que existe una relación directa entre el índice de gravedad y el esfuerzo terapéutico y el padecimiento del síndrome ${ }^{(40)}$. No obstante, hay que señalar que también existen investigaciones en las que no se han encontrado diferencias estadísticamente significativas en el padecimiento de este síndrome y el personal que trabaja en servicios especiales ${ }^{(41)}$. En relación a este dato, existe un estudio llevado a cabo en 59 hospitales españoles de más de 150 camas, donde se evidenciaban niveles más bajos de burnout en el personal que trabajaba en áreas mas especializadas que en unidades de hospitalización (42). Podría considerarse explicación a ello, que en el caso del Área de Ictus, los pacientes que ingresan pasan, en general, un periodo "critico" de unos 3 días, en los que después de ser evaluada su situación son derivados a otros centros o incluso a su domicilio, ocurriendo estancias más largas en otro tipo de servicios no tan especializados.

\section{CONCLUSIONES.}

En conclusión se puede afirmar que el Síndrome del Burnout está presente en el $31,81 \%$ de esta población y como tal podría ser una amenaza en la calidad de servicios prestados y el bienestar del personal enfermero. No obstante sería conveniente considerar como posibles líneas futuras de investigación ampliar el presente estudio en otras unidades del Hospital.

Por otro lado, es importante considerar el resto de resultados de las subescalas puesto que existen porcentajes altos de personas que se encuentran con niveles que se corresponden con tendencias al padecimiento del síndrome.

Aunque en este estudio no se han encontrado diferencias estadísticamente significativas en cuanto al padecimiento del síndrome y las diferentes variables a estudio, es necesario seguir investigando sobre factores sociodemográficos que pudieran influir en su aparición, para desarrollar acciones preventivas o de tratamiento acordes con dichos factores.

Se debería seguir estudiando el fenómeno Síndrome del Burnout en esta población y otras, a la vez que se trabaje en estrategias y tratamiento del estrés laboral crónico adaptados a las características de cada servicio, a fin de prevenir y tratar la aparición del síndrome en el personal de enfermería mediante programas de formación 
relacionados con adquisición de habilidades sociales, habilidades de comunicación ${ }^{(43)}$ manejo de la autoestima, técnicas de relajación, técnicas de afrontamiento, etc. (37) Esta labor, sin duda, mejoraría la calidad de vida laboral del personal y en consecuencia aumentaría el compromiso profesional y la mejora de los cuidados prestados a los pacientes.

\section{REFERENCIAS}

1. Luceño Moreno L, Martín García J, Rubio Valdehita S, Díaz Ramiro E. Factores psicosociales en el entorno laboral, estrés y enfermedad.EduPsykhé. 2004; 3(1):95108.

2. Frade Mera MJ, Vinagre Gaspar R, Zaragoza García I, Viñas Sánchez S, Antúnez Melero E, Álvarez González $S$ et al. Síndrome de Burnout en distintas unidades de cuidados intensivos. Enferm Intensiva. 2009; 20(4):131-140.

3. Muñoz MJ, Pérez MI, Granero J. Inmunidad de enfermería a los efectos del Burnout. Metas de Enferm.2002; 51(6):6-9.

4. García Grau N, Carmona Heredia A, Roca Biosca A, Olona Cabases M. Síndrome de Burnout en los equipos de enfermería de Cuidados Intensivos de Cataluña. Metas de Enferm. 2004; 7(2):6-12.

5. Freudemberguer HJ. Staff burn-out. Journal of social issues.1974; 30: 159-165.

6. Gálvez M, Moreno B, Mingote JC. El desgaste profesional del médico. Revisión y guías de buenas prácticas. El vuelo de Ícaro. Madrid: Díaz de Santos; 2009.

7. Maslach C, Jackson S. The measurement of experienced Burnout. J Occup Behavior. 1981; 2:99-113.

8. Maslach C, Jackson S, Leiter Y. Maslach Burnout Inventory. Manual.3ed.Palo alto. California: Consulting psychology Press; 1996.

9. Méndez Y. Estrés laboral o síndrome de "Burnout". Acta Pediatr Mex.2004; 25(5):299-302.

10. Gil Monte P, Peiró JM. Perspectivas teóricas y modelos interpretativos para el estudio del síndrome de quemarse en el trabajo. Anales de psicología. 1999; 15(2):261-268.

11. Gil Monte P. El síndrome de quemarse por el trabajo (Síndrome de Burnout) en profesionales de enfermería. Revista electrónica InteracaoPsy. 2003; 1(1):19-33.

12. Artiga Ruiz A. La enfermería ante el síndrome del Burnout. Estudio comparativo entre atención primaria y atención especializada. RevEnf Científica. 2000; 220221:11-16.

13. Burgos Díez $P$, Ruiz Albi T, QueipoBurón D, Rescalvo Santiago F, Martínez León MM, del Amo Merino $P$, et al. Calidad de vida relacionada con la salud en trabajadores sanitarios. Med Segur Trab. 2012; 58(226):27-34.

14. Cano MA, GarcíaGarcía $E$, López M, Parera N. ¿Está quemada la enfermería?: síndrome de burnout. Enferm Intensiva.1996; 7(4):138-146.

15. Bustinza Arriortua A, López- Herce J, Carrillo Álvarez A, Vigil Escribano MD, De Lucas García N, Panadero Carlavilla E. Situación de Burnout de los pediatras intensivistas españoles. An Pediatr. 2000; 52(5):418-423.

16. Gil Monge PR, Moreno Jiménez B. El síndrome de quemarse por el trabajo (Burnout). Grupos profesionales de riesgo. Madrid: Pirámide; 2007.

17. Da Silva H, Vega ED, Pérez A. Estudio del síndrome de desgaste profesional entre los profesionales sanitarios de un hospital general. Actas Esp. Psiquiatri.1999; 27:310-320. 
18. Faura T, Roige M, Serra R, Ortega C, Monegal M, Roige A, et al. Prevalencia del Síndrome de Burnout en enfermeras de hospitalización y de Atención Primaria. EnfermClin.1995; 5:105-110.

19. Ribera Domene D, Cartagena de la Peña E, ReigFerrer A, Roma Ferri MT, Sans Quintero I, CaruanaVañó A. Estrés laboral y salud en los profesionales de enfermería. Estudio empírico en la provincia de Alicante. Alicante: Secretariado de publicaciones, Universidad de Alicante; 1993.

20. Quintanilla M. Prevalencia del síndrome del Burnout en las enfermeras de la unidad de paciente crítico del hospital del trabajador Santiago de Chile y una propuesta de intervención. RevChilMedlnten. 2004; 19(1): 33-37.

21. Solano MC, Hernández $P$, Vizcaya MF, Reig A. Síndrome de Burnout en profesionales de enfermería de cuidados críticos. Enferm Intensiva. 2002; 13(1):9-16.

22. Molina JM, Abalos F, Jiménez I. Burnout en enfermería y atención hospitalaria. EnfermClin.2005; 15(5):275-282.

23. Ibañez N, Vilaregut A, Abio A. Prevalencia del Síndrome de Burnout y el estado general de salud en un grupo de enfermeras de un hospital de Barcelona. EnfermClin.2004; 14(3):42-51.

24. Tomás Sábado J, MaynegreSantaulària $M$, Pérez Bartolomé $M$, Alsina Rodríguez M, Quinta Barbero R, Granell Navas S. Síndrome de burnout y riesgo suicida en enfermeras de atención primaria. EnfermClin. 2010; 20(3):173-178.

25. Atance Martínez JC. Aspectos epidemiológicos del Síndrome del Burnout en personal sanitario. RevEsp Salud Pública. 1997; 71:293-303.

26. Martínez García M, Centeno Cortés C, Sanz Rubiales A, Del Valle ML. Estudio sobre el Síndrome de Burnout en Profesionales de Enfermería de Cuidados Paliativos del País Vasco. RevMedUniv Navarra.2009; 53(1): 3-8.

27. Seisdedos N. Manual MBI, Inventario Burnout de Maslach. Madrid: TEA; 1997.

28. Albaladejo $R$, Villanueva $R$, Ortega $P$, Astasio $P$, Calle ME, Dominguez V. Síndrome del Burnout en el personal de enfermería de un hospital de Madrid. RevEsp Salud Pública.2004; 78(4):505-516.

29. Sanchez Alonso P, Sierra Ortega VM. Sindrome de Burnout en el personal de enferemria en UVI. Enf Global. 2014; 13(1): 252-266.

30. Tuesca Molina R, Iguarán Urdaneta $M$, Suárez Lafaurie M, Vargas Torres G, Vergara Serpa D. Síndrome de desgaste profesional en enfermeros/as del área metropolitana de Barranquilla. SaludUninorte.2006; 22(2):84-91.

31. Aragón Paredes MB, Morazán Pereira DI, Pérez Montiel R. Síndrome de Burnout en médicos y personal de enfermería del Hospital Escuela "Oscar Danilo Rosales Arguello, León, 2007.Universitas.2008; 2:33-38.

32. Ríos Risquez MI, Peñalver Hernández F, Godoy Fernández C. Burnout y salud percibida en profesionales de enfermería de cuidados intensivos. Enferm Intensiva.2008; 19(4):169-178.

33. Puialto Durán MJ, Antolín Rodríguez R, Moure Fernández L. Prevalencia del síndrome del quemad@ y estudio de factores relacionados en I@s enfermer@as del CHUVI (Complejo Hospitalario Universitario de Vigo). Enf Global. 2006; 5(1):1-18.

34. Rodríguez López M, Fernández Barral R, Benítez Canosa MC, Castiñeira MJ, Brea Fernández AJ. Correlación entre carga de trabajo, síndrome de Burnout y calidad de vida en una unidad de críticos. Enf Global. 2008; 7(3): 1-11.

35. Benito Aracil L, Puig Llobet M, LluchCanut T. Burnout en la profesión enfermera: una aproximación bibliométrica inicial. Metas de Enferm. 2013; 16(6): 68-72.

36. Escobar Aguilar G, Gómez García T, Ignacio García E, Rodríguez Escobar J, Moreno Casbas T, Fuentelsaz Gallego C, et al. Entorno laboral y seguridad del paciente: comparación de datos entre los estudios SENECA y RN4CAST. EnfermClin. 2013; 23(3): 103-113. 
37. González Correales R. Síndrome de Burnout: una oportunidad para aprender y reflexionar. Semergen. 2003; 29:2-3.

38. Alvarez Verdugo LP, Prieto Bocanegra BM. Prevalencia de desgaste profesional en personal de enfermería de un hospital de tercer nivel de Boyacá, Colombia. Enf Global. 2013; 12(1): 73-88.

39. Bacaicoa Parrado P, Díaz Herrera V, Gea Valero M, Linares Pérez J, Araya Pérez E, Alba Domínguez Juan F, et al. Comparativa del síndrome burnout entre el personal de enfermería en cardiología de dos hospitales de tercer nivel. EnfermCardiol. 2012; 19(55-56):34-40.

40. Romero Massa E, Lorduy Bolívar JP, Pájaro Melgar C, Pérez Duque CA. Relación entre la carga laboral de enfermería y la gravedad del paciente en unidades de cuidado intensivo de adultos.Aquichan. 2011; 11(2): 173-186.

41. Esquivel Morocho A. Burnout en personal sanitario del Hospital U.y P. "La Fe". [trabajo fin de master]. Universidad Cardenal Herrera, Valencia; 2013.

42. Fuentelsaz Gallego C, Moreno Casbas T, Gómez García, González María E. Entorno laboral, satisfacción y burnout de las enfermeras de unidades de cuidados críticos y unidades de hospitalización. Proyecto RN4CAST-España. Enferm Intensiva. 2013; 24 (3): 104-112.

43. García Moyano L, Arrazola Alberdi O, Barrio Forné N, Grau Salamero L. Habilidades de comunicación como herramienta útil para la prevención del síndrome de burnout. Metas Enferm. 2013; 16(1):57-60. 